\title{
Coronavirus Disease 2019: A Review of Transmission, Clinical Manifestations, Laboratory, and Radiologic Findings
}

\section{Sabah Mohamed Alharazy*}

Independent Researcher, Malaysia

*Corresponding Author: Sabah Mohamed Alharazy, Independent Researcher, Malaysia.
Received: March 01, 2021

Published: April 27, 2021

(C) All rights are reserved by Sabah Mohamed Alharazy.

\begin{abstract}
Coronavirus Disease 2019 (COVID-19) is a highly transmissible and pathogenic viral infection caused by severe acute respiratory syndrome coronavirus-2 (SARS-CoV-2). It has been confirmed that COVID-19 is transmitted from person to person through infected droplets or direct contact. The symptoms of COVID-19 range from asymptomatic or mild infection to critical illness. Symptoms may include fever, cough, fatigue, dyspnea, myalgia, headache, sore throat, nasal congestion, loss of smell or taste. Gastrointestinal symptoms, such as nausea, vomiting, anorexia, and diarrhea can also occur. Ground-glass opacity (GG0) with or without consolidation is the hallmark of this disease in Chest Computed Tomography. Many laboratory parameters are affected in COVID-19 patients, some of which are associated with disease severity and poor prognosis, such as severe lymphopenia, abnormal coagulation parameters, and elevated levels of D-dimer, ferritin, and C-reactive protein (CRP). This review summarizes the current understanding of transmission, common clinical features, common laboratory as well as radiologic findings of COVID-19 infection.
\end{abstract}

Keywords: Coronavirus Disease 2019 (COVID-19); Severe Acute Respiratory Syndrome Coronavirus-2; Transmission; Clinical Features; Laboratory Parameters; Radiologic Findings

\section{Introduction}

Coronavirus Disease 2019 (COVID-19) is caused by the virus known as severe acute respiratory syndrome coronavirus 2 (SARSCoV-2) [1], which was first reported in Wuhan, China, at the end of 2019 [2] and has since spread widely throughout the world. As of 14 December 2020, COVID-19 affects 218 countries and territories across the world and 2 international conveyances. The total confirmed cases of COVID-19 are 72,654,602, with a total reported death of 1,619,028. Approximately 50,871,457 individuals have now recovered from this virus worldwide [3]. This review provides the current understanding of the mode of transmission, as well as the common clinical manifestations, laboratory, and radiologic findings of this disease.

\section{Modes of COVID-19 transmission}

COVID-19 infection is mainly transmitted through respiratory droplets of infected individuals. The modes of transmission of COVID-19 infection are included in table 1 [4-6].
Incubation period of COVID-19

The incubation period is important for understanding the nature of the spread of the COVID-19 epidemic as well as the effective length of the quarantine period. This is defined as the time between the date of infection and the onset of the disease. The WHO estimated the median incubation period of this infection to be between 5 to 6 days but may be up to 14 days [4,7]. However, a large cross-sectional study including 1,084 cases with confirmed COVID-19 from Wuhan found an incubation period of more than 14 days in $5 \%\left(95^{\text {th }}\right.$ percentile, 16.32$)$ to $10 \%$ of cases $\left(90^{\text {th }}\right.$ percentile, 14.28) and more than 20 days (99th percentile, 20.31) in $1 \%$ of cases [8]. The median of the incubation period was 7.76 days, while the mean was 8.29 days [8]. The study used a renewal process (renewal theory in probability) [8].

\section{Clinical manifestations of COVID 19 infection}

COVID 19 infection has a wide range of clinical manifestations, ranging from asymptomatic or mild manifestations to critical ill- 


\begin{tabular}{|c|c|}
\hline Modes of COVID-19 transmission & The properties of this Mode \\
\hline roplet transmission & $\begin{array}{l}\text { Respiratory droplet transmission is the predominant mode of transmission of COVID-19 in- } \\
\text { fection. } \\
\text { The transmission of the virus occurs when the virus spreads from infected individuals while } \\
\text { sneezing, } \\
\text { coughing or talking without covering the mouth or nose. } \\
\text { Individuals who are in close contact with an infected person may become infected when these } \\
\text { infectious respiratory droplets get into their mouth, nose, or eyes. } \\
\text { Respiratory droplets are generally > 5-10 } \mu \text { m in diameter. } \\
\text { "Close contact" is described in various ways: } \\
\text { The WHO described it as within } 1 \text { metre. } \\
\text { The U.S. CDC describes it as "within } 6 \text { feet (1.8 m) of the infected person of } 15 \text { minutes or } \\
\text { more over a } 24 \text {-hour period starting from } 2 \text { days' prior illness onset (or, for asymptomatic } \\
\text { patients, } 2 \text { days before to test specimen collection) until the time the patient is isolated. } \\
\text { The ECDC suggests that close proximity is typically less than } 1 \text { meter ( } 3.3 \mathrm{ft} \text { ) apart. }\end{array}$ \\
\hline $\begin{array}{c}\text { airborne transmission/ Aerosol } \\
\text { transmission }\end{array}$ & $\begin{array}{l}\text { Airborne transmission is not a common mode of transmission of COVID-19 infection, it re- } \\
\text { mains controversial outside medical facilities. } \\
\text { The transmission of the virus occurs by aerosols, the dissemination of a droplet nuclei that } \\
\text { capable of being suspended in the air over long distances and for longer periods of time. } \\
\text { Aerosols or droplet nuclei refer to droplets with a diameter of } \leq 5 \mu \mathrm{m} \text {. } \\
\text { Airborne transmission can occur in healthcare settings, with certain aerosol-generating } \\
\text { medical procedures performed on COVID-19 patients. } \\
\text { Outside of medical facilities, it occurs in enclosed space, crowded and poorly ventilated areas } \\
\text { According to US. CDC, in some cases, there is evidence that individuals with COVID-19 appear } \\
\text { to have spread the infection to others who were more than } 6 \text { feet away. } \\
\text { This form of transmissions occurred within the confined areas, that under ventilated where } \\
\text { infected person could have breathed heavily, for example when singing or exercising. }\end{array}$ \\
\hline Physical intimacy & $\begin{array}{l}\text { It carries a substantial risk of transmission due to close proximity. } \\
\text { The virus spreads through saliva, mucus, and kissing. }\end{array}$ \\
\hline $\begin{array}{l}\text { Vertical transmission (mother to } \\
\text { child) }\end{array}$ & $\begin{array}{l}\text { Intrauterine transmission has been reported in a few cases but is generally rare. } \\
\text { There is insufficient evidence to ensure the vertical transmission of COVID- } 19 \text { through breast } \\
\text { feeding, as no viable breast milk virus has been reported in the studies. } \\
\text { WHO recommends that mothers with suspected or confirmed COVID-19 should be advised to } \\
\text { start or continue breastfeeding. }\end{array}$ \\
\hline
\end{tabular}




\begin{tabular}{|c|c|}
\hline $\begin{array}{l}\text { Bloodborne } \\
\text { transmission }\end{array}$ & $\begin{array}{l}\text { The role of bloodborne transmission remains unclear, and low plasma and serum viral titers } \\
\text { indicate that there may be a low risk of transmission via this route. }\end{array}$ \\
\hline Fecal/urine-oral transmission & No significant evidence of COVID-19 virus transmission through feces and urine. \\
\hline Fomite transmission. & $\begin{array}{l}\text { Fomite transmission is considered a likely mode of transmission for COVID-19 infection. } \\
\text { Despite consistent evidence of surface contamination by SARS-CoV-2 and the survival of } \\
\text { the virus on certain surfaces, no specific reports have explicitly shown fomite transmission. } \\
\text { People who have made contact with infectious surfaces also have close contact with an infec- } \\
\text { tious individual, making it difficult to distinguish between respiratory droplets and fomite } \\
\text { transmission. }\end{array}$ \\
\hline $\begin{array}{l}\text { Abbreviation } \\
\text { WHO: World Health Organization; } \\
\text { CDC: Centres for Disease Control and } \\
\text { Prevention; ECDC: European Center } \\
\text { for Disease Prevention and Control. }\end{array}$ & $\begin{array}{l}\text { Table } 1 \text { adapted from: World Health Organization (WHO) 2020: Transmission of SARS-CoV-2: } \\
\text { implications for infection prevention precautions. [4]; European Center for Disease Preven- } \\
\text { tion and Control (ECDC) 2020: "Q and A on COVID-19: Basic facts” [5]. Centers for Disease } \\
\text { Control and Prevention (CDC 2020) How COVID-19 Spreads [6]. }\end{array}$ \\
\hline
\end{tabular}

Table 1: Modes of COVID-19 transmission [4-6].

ness $[9,10]$. The majority of COVID-19 infections are not severe [7,11-13]. Evidence of COVID-19 transmission from asymptomatic and presymptomatic patients has been reported [4,5]. However, the exact proportion of the transmission due to pre-symptomatic or asymptomatic infection compared to symptomatic infection remains unclear [14].

COVID-19 is most commonly associated with respiratory symptoms, but it can also cause several extra-pulmonary manifestations [15]. The COVID-19 associated symptoms and their frequencies are summarized in table 2 based on the study of 1,099 patients from China [16] and the WHO-China Joint Mission report of 55,924 patients with COVID-19 [7]. Symptoms were also summarized from Surveillance data of 1,320,488 patients from the United States [17] and meta-analysis including 24,410 patients with COVID-19 from 9 countries (China, the United Kingdom, the United States, Singapore, Italy, Australia, Japan, Korea, and the Netherlands) [18]. Of note, the accurate frequency of COVID-19 associated symptoms remains unknown.

Similarly, to the studies summarized in table 2, two large systematic reviews of COVID-19 patients $(11,028$ patients in the first review and 41,409 patients in the second review) from different countries found that fever $(58.66 \%-72.4 \%)$ and cough $(54.52 \%-$ $55.5 \%)$ are the most frequently reported symptoms $[9,19]$. Other 


\begin{tabular}{|c|c|c|c|c|}
\hline Study & $\begin{array}{l}\text { Guan et al } \\
\text { study }[16]\end{array}$ & $\begin{array}{c}\text { WHO-China } \\
\text { Joint Mission } \\
{[7]}\end{array}$ & $\begin{array}{l}\text { Stokes EK, } \\
\text { study [17] }\end{array}$ & A systematic review and meta-analysis [18] \\
\hline Patients no & 1,099 & 55,924 & $1,320,488$ & 24,410 \\
\hline Median/mean age (years) & 47 years & 51 years & 48 years & 49 years \\
\hline \multicolumn{5}{|l|}{ Symptoms (\%) } \\
\hline Fatigue & $38.1 \%$ & $38.1 \%$ & NA & $31 \%$ \\
\hline Dyspnea & $18.7 \%$ & $18.6 \%$ & $28.5 \%$ & $23 \%$ \\
\hline Sputum production & $33.7 \%$ & $33.4 \%$ & NA & NA \\
\hline Myalgia or arthralgia & $14.9 \%$ & $14.8 \%$ & $36.1 \%$ & $17 \%$ \\
\hline $\begin{array}{c}\text { Nasal congestion and/or } \\
\text { Rhinorrhea }\end{array}$ & $4.8 \%$ & $4.8 \%$ & $6.1 \%$ & $5 \%$ \\
\hline $\begin{array}{c}\text { Conjunctival congestion/ } \\
\text { conjunctivitis }\end{array}$ & $0.8 \%$ & $0.8 \%$ & NA & $2 \%$ \\
\hline Haemoptysis & $0.9 \%$ & $0.9 \%$ & NA & $2 \%$ \\
\hline Nausea and vomiting & $5 \%$ & $5 \%$ & $11.5 \%$ & $10 \%$ \\
\hline Diarrhea & $3.8 \%$ & $3.7 \%$ & $19.3 \%$ & $10 \%$ \\
\hline loss of smell or taste. & NA & NA & $8 \%$ & NA \\
\hline NA; not available & & & & \\
\hline
\end{tabular}

Table 2: Clinical Manifestations of COVID 19 infection [7,16-18]

common symptoms described in the first review were sore throat (16.2\%), dyspnea (8.8\%), and muscle pain (22.1\%). Headache, rhinorrhea and diarrhea were also reported in $10.5 \%, 9.2 \%$, and $7.9 \%$ of patients, respectively [19]. Other frequent symptoms reported in the second review were dyspnea (30.82\%), malaise $(29.75 \%)$, fatigue $(28.16 \%)$, and sputum/secretion (25.33\%). Neurologic symptoms (20.82\%), dermatologic manifestations (20.45\%), anorexia (20.26\%), myalgia (16.9\%), sneezing (14.71\%), sore throat (14.41\%), rhinitis (14.29\%), goosebumps (13.49\%), headache (12.17\%), chest pain (11.49\%), and diarrhea (9.59\%) were also reported. The least frequent symptom was hemoptysis $(1.65 \%)$ [9].
Although fever is the most commonly reported symptom in patients with COVID-19 [7,9,16,18-21], the absence of fever at the time of initial presentation does not exclude COVID-19. In a large case series study involving 5,700 hospitalized patients with confirmed COVID-19 in New York City, the United States, fever with a temperature of $>38^{\circ} \mathrm{C}$ was reported in only $30.7 \%$ of patients in the admission [22]. In the previous surveillance data submitted to CDC, the United States, including 1,320,488 confirmed COVID-19 cases, fever which either subjective or with a temperature $>38^{\circ} \mathrm{C}$ was reported in only $43.1 \%$ of cases [17]. In a retrospective cohort study, Emergency Medical Services (EMS) were involved in 124 of 
775 cases of COVID-19 (16.0\%) [23]. Based on the EMS assessment, 43 of 147 encounters (29.3\%) had no fever, cough, or dyspnea, and based on the clinical examination, fever, tachypnea, or hypoxia were reported in 43 of 84 encounters (51.2\%), 42 of 131 (32.1\%), and 60 of 112 (53.6\%), respectively [23]. It is therefore, possible to miss afebrile patient if surveillance depends on the detection of fever in the case definition of COVID-19 [23]. Similarly, in the previous Chinese case series of 1,099 patients, fever was defined as an axillary temperature of $37.5^{\circ} \mathrm{C}$ or higher was reported only in $43.8 \%$ of patients on admission and in $88.7 \%$ of patients on hospitalization [16].

In some studies, in patients with COVID-19, fever has been reported to be at least $37.3^{\circ} \mathrm{C}$ and any temperature higher. Notably, the proportion of patients with a temperature between $38.1^{\circ} \mathrm{C}$ and $39^{\circ} \mathrm{C}$ appears to be higher than those with fever with temperatures between $37.3^{\circ} \mathrm{C}$ and $38^{\circ} \mathrm{C}$ or above $39^{\circ} \mathrm{C}$. In one study of 21 patients with COVID-19 infection, fever was identified as high fever (the temperature above $39^{\circ} \mathrm{C}$ ), moderate fever (the temperature ranged between $38.1^{\circ} \mathrm{C}$ and $39^{\circ} \mathrm{C}$ ), and low-grade fever (the temperature ranged between $37.5^{\circ} \mathrm{C}$ and $38^{\circ} \mathrm{C}$ ) in $24 \%, 33 \%$, and $29 \%$, of patients, respectively [24]. In another study of 137 patients with COVID-19, the majority of patients $(81.8 \%)$ had a fever on presentation, approximately $31.3 \%$ had a moderate fever (the temperature ranged between $38.1^{\circ} \mathrm{C}$ and $39^{\circ} \mathrm{C}$ ), $25 \%$ had a low fever (the temperature ranged between $37.3^{\circ} \mathrm{C}$ and $38^{\circ} \mathrm{C}$ ), and only $17.9 \%$ had a fever with a temperature above $39^{\circ} \mathrm{C}$. By contrast, $25 \%$ of patients did not experience fever on presentation [25]. Similarly, in another study of 41 patients, almost all patients (98\%) developed fever; the majority (44\%) had a moderate fever (the temperature ranged between $38.1^{\circ} \mathrm{C}$ and $39^{\circ} \mathrm{C}$ ), $34 \%$ had a high fever with a temperature above $39^{\circ} \mathrm{C}$, and $20 \%$ had a low-grade fever (the temperature ranged between $37 \cdot 3^{\circ} \mathrm{C}-38^{\circ} \mathrm{C}$ ) [20]. The degree of fever did not differ significantly between the ICU and non-ICU patients. A higher proportion of ICU patients (77\%) had a fever with a temperature higher than $38.1^{\circ} \mathrm{C}$, and $23 \%$ had a low-grade fever. In contrast, $79 \%$ of non-ICU patients had a fever with a temperature above $38.1^{\circ} \mathrm{C}$, and $18 \%$ had a low-grade fever [20]. In a study of 221 patients with COVID-19, most patients (90.5\%) developed a fever on presentation; $47.1 \%$ had a fever with a temperature ranged between $38.1^{\circ} \mathrm{C}$ and $39^{\circ} \mathrm{C}, 25.8 \%$ had a fever with a temperature ranged between $37.3^{\circ} \mathrm{C}$ and $38^{\circ} \mathrm{C}$ and only $17.6 \%$ had a fever with a temperature above $39^{\circ} \mathrm{C}$ [26]. A higher proportion of severely affected patients experienced fever with a temperature $>38^{\circ} \mathrm{C}$ compared with non-severely affected patients, (49 [89.1\%] versus 94 [56.6\%]; P < 0.001) [26]. Similarly, in the previous Chinese case series of 1,099 patients, fever with a temperature of $>$ $38^{\circ} \mathrm{C}$ was more prevalent in severely ill patients $(68.4 \%)$ than in those with mild conditions (57.4\%) during hospitalization [16].

The estimated median duration of fever in 94.3\% (235/249) febrile patients with COVID-19 was 10 days ( 95 Confidence Interval [CI]: 8-11 days) after the onset of symptoms [27]. Those patients transferred to the ICU had a significantly longer duration of fever than those who were not in the ICU (31 days versus 9 days after symptoms onset, respectively, $\mathrm{P}<0.0001$ ) [27]. By the resolution of fever, half of the patients had reverse-transcription polymerase chain reaction (RT-PCR) negative with their upper respiratory tract samples and $94.5 \%$ of patients showed radiologic improvement [27].

Fever may also not be present in the elderly or may occur later in the course of the disease [28]. It has been reported that fever may be blunt or even absent in elderly patients with bacterial or viral infections due to low basal temperature and aging disturbances in thermal homeostasis, as well as frequent use of drugs such as aspirin [29]. In addition to the absence of fever, elderly and immunosuppressed patients may experience atypical symptoms such as fatigue, decreased alertness, reduced mobility, diarrhea, a loss of appetite, and delirium [30].

The cough is the second most frequently reported symptom in COVID-19 patients [7,9,16,18-21]. Among survivors with COVID-19 (137 out of 191), the median duration of fever was 12 days (Interquartile range [IQR] 8-13) and the cough continued for 19 days (IQR 12-23) [31].

Dyspnea is more frequently reported in COVID-19 patients with severe disease $[11,20]$. The median time from the illness onset to the dyspnea was 5 to 8 days [11,20]. A study of 1,590 patients with COVID-19 showed that dyspnea was related to an approximately two-fold risk of critical disease (Odds Ratio (OR): 1.88; 95\% CI, 1.18-3.01; $\mathrm{P}=0.01$ ) [32]. During a prospective analysis of 1,045 hospitalized patients with COVID-19 in North-Eastern France, dyspnea at admission was related to severe disease (OR: 2.5; 95\% CrI: 1.83.4) and death (OR: 2.1; 95\% CrI: 1.2-3.4) [33].

Gastrointestinal symptoms like nausea or vomiting and diarrhea were uncommon (Table 2) [7,16-18], however, some patients 
had predominantly gastrointestinal symptoms at the onset of the illness. During a study of 204 patients with COVID-19, almost half of patients $(50.5 \%)$ presented with gastrointestinal symptoms including anorexia (78.6\%), vomiting (3.9\%), diarrhea (34\%), and abdominal pain (1.9\%) [34]. Compared with patients without gastrointestinal symptoms, those presenting with gastrointestinal symptoms had an extended time from the onset to the admission and a worse prognosis (9.0 days versus 7.3 days) [34]. Notably, in $3 \%$ of cases, there were gastrointestinal symptoms but no respiratory symptoms [34]. Patients with gastrointestinal symptoms had higher mean liver enzyme levels, a lower monocyte count, and a longer prothrombin time than those without digestive symptoms [34]. In another study of 393 patients with COVID-19 in New York, the United States, the manifestations of the disease were generally almost like those within the previous large case series of 1,099 patients in China [16], however, gastrointestinal symptoms were more frequent. Diarrhea and nausea/vomiting were reported in 23.7 and 19.1 percent, respectively, while those symptoms occurred only in 3.8 to 5 percent of patients in China [35]. This discrepancy may indicate geographic variation or differential reporting [35]. Another study of 59 patients with COVID-19 infection in Hong Kong showed that the prevalence of gastrointestinal symptoms (including nausea/vomiting, diarrhea, and abdominal pain/discomfort) was $25.4 \%$, and the stool viral RNA was found in $38.5 \%$, and $8.7 \%$ among those with and without diarrhea respectively [36].

Loss of smell or taste (e.g., anosmia and dysgeusia) have also been reported $[17,37]$, and may precede the onset of other symptoms [38], particularly among women and young or middle-aged patients who do not need hospitalization [38,39]. A large study of 2,013 European patients with mild to moderate COVID-19 found that 1,754 patients $(87 \%)$ reported a loss of smell, while 1136 $(56 \%)$ reported taste dysfunction. The mean duration of olfactory dysfunction was 8.4 days [40]. The study showed that anosmia may occur in the absence of nasal obstruction or inflammation. Only two-thirds of patients who reported olfactory symptoms and performed an olfactory examination were found to have abnormal results [40]. A systematic review and meta-analysis of 10 studies involving 1,627 patients, across 9 countries (Italy, France, the United States, Spain, Iran, China, Belgium, the United Kingdom, and Mexico) investigating loss of smell and/or taste with COVID-19 infection reported a pooled prevalence of $52.7 \%$ (95\% CI, 29.64\%$75.23 \%)$ and $43.9 \%$ (95\% CI, 20.46\%-68.95\%) of olfactory and gustatory dysfunction respectively [37].The underlying pathogenetic mechanism of taste and olfactory disorders in COVID-19 in- fection may be explained by the high expression of the ACE2 receptor of SARS-CoV-2 on the epithelial cells of the oral cavity mucosa [41].

Most children with COVID-19 are asymptomatic or have mild symptoms [42]. However, some children may have a severe illness and a fatal outcome [42]. Fever and cough are the most common symptoms of COVID 19 in children [42]. Data from 18 systematic reviews show that $15-42 \%$ of COVID-19 children and adolescents were asymptomatic and the most common symptoms were fever (46\% to $64.2 \%$ ) and cough (32\% to 559\%) [43]. All the other symptoms or signs like rhinorrhoea, sore throat, headache, fatigue/ myalgia, and gastrointestinal symptoms such as diarrhea and vomiting were uncommon, occurring at less than 10\%-20\% [43]. A literature review of COVID-19 infection in neonates and children has shown that some clinical features, such as increased incidence of fever, vomiting, and diarrhea, as well as longer incubation periods, were more common in children than in adults [44]. Another systematic review of 7,480 children with clinical findings that available in 1,780 children aged 1 month to 18 years with confirmed COVID-19 showed that the most commonly reported symptoms were fever (51.6\%), cough (47.3\%), and sore throat (17.9\%) [45]. Approximately, $7.7 \%$ of patients developed dyspnea, and $3.3 \%$ required oxygen supplementation for hypoxia [45]. In contrast to adults, children are more likely to develop extra-respiratory symptoms, particularly diarrhea (9.4\%) and vomiting (7.3\%) [45].

In addition to the gastrointestinal symptoms, COVID-19 can cause several extra-pulmonary manifestations, including thrombotic complications, myocardial dysfunction and arrhythmia, acute coronary syndromes, acute kidney injury, hepatocellular injury, hyperglycemia, and ketosis, ocular symptoms, neurologic symptoms, and dermatologic manifestations [15]. A comprehensive review of all COVID-19 extra-respiratory manifestations is beyond the scope of this review.

\section{Laboratory features of COVID-19}

The most-reported laboratory findings in the first large study of 1,099 COVID-19 patients from China were lymphopenia (83.2\%), leukopenia (33.7\%), and thrombocytopenia (36.2\%) [16]. Most of the patients $(60.7 \%)$ had an elevated level of C-reactive protein (CRP) [16]. Increased levels of alanine aminotransferase, aspartate aminotransferase, creatine kinase, and D-dimer were less common. These findings are more prominent in patients with severe disease than in patients with non-severe disease $(96.1 \%$ versus $80.4 \%$ for lymphopenia, $57.7 \%$ versus $31.6 \%$ for thrombocytopenia, and $61.1 \%$ versus $28.1 \%$ for leukopenia) [16]. 
Leukopenia appears to be inconsistent, as several studies have shown that patients with COVID-19 infection have varying white blood cell counts $[11,20,21]$. In a study of 41 patients with COVID-19 infection, $45.0 \%$ of patients had normal leukocyte counts (white blood cell count: 4000-10000 per $\mu \mathrm{L}$ ), 25\% had leukopenia (white blood cell count $<4000$ per $\mu \mathrm{L}$ ) with the majority $(63.0 \%$ ) had lymphopenia while $30.0 \%$ had leukocytosis (>10000 per $\mu \mathrm{L}$ ) [20]. Patients with severe disease were found to have leukocytosis (2.0-folds increase) than those with non-severe disease [20]. Similarly, in a study of 140 patients with COVID-19 infection, most of the patients (68.1\%) had normal leukocyte counts, $19.6 \%$ had leukopenia with $75.4 \%$ of the patients had lymphopenia and $12.3 \%$ had leucocytosis [46]. Notably, leukocytosis was significantly found in severe compared to non-severe patients [46]. It was also reported that non-survivors were more likely to develop leucocytosis [11].

Lymphopenia is a common reported haematologic finding in COVID-19 patients $[11,16,20,21]$. It is also more prominent among patients with severe compared to non-severe COVID-19 disease $[16,21,33]$. Furthermore, severe lymphopenia has been associated with critical illness and mortality in COVID-19 [13,33]. Among 24 critically ill patients with confirmed COVID-19 who were admitted to ICUs in the Seattle region, lymphopenia was reported in $75 \%$ of patients on admission [13]. In another study of 1,045 hospitalized COVID-19 patients from France, lymphopenia was observed in $60.9 \%$ of patients and a lymphocyte count $<1,000$ per $\mu \mathrm{L}$ (OR: 1.4; 95\% CrI: 1.1-2.0) was associated with the development of severe disease [33]. A systematic review and meta-analysis of 24 studies involving 3,099 patients, found that lymphopenia with the cut-off point at $\leq 1100$ per $\mu \mathrm{L}$ was associated with the threefold risk of poor outcome in COVID-19 patients [47]. The correlation appeared greater in younger patients compared to older patients [47]. Because the lymphocytes have ACE2 receptors on their surface, the SARS-CoV-2 can directly infect these cells and causing their lysis [48]. Other potential explanations for lymphopenia in COVID-19, including lymphocyte apoptosis induced by a cytokine storm [48]. Atrophy of lymphoid organs, including the spleen, and further impairs the turnover of lymphocytes [48]. Inhibiting the proliferation of lymphocytes by coexisting lactic acidosis, which may be more common among cancer patients at increased risk for complications from COVID-19 [48].

Neutrophilia (increased neutrophil counts) and increased NLR (neutrophil to lymphocyte) ratio were also reported in COVID-19 patients [11]. NLR is determined by dividing the absolute neutro- phil count by the absolute lymphocyte count and used as a measure of systemic inflammation and infection [49]. Additionally, elevated NLR is a risk factor of mortality in malignancy, acute coronary syndrome, intracerebral hemorrhage, polymyositis, and dermatomyositis $[11,49]$.

Thrombocytopenia (low platelet count) is also frequently present in COVID-19 and has been associated with poor outcomes [12]. The possible mechanism of thrombocytopenia in COVID-19 includes purely consumptive, in particular, endothelial damage, platelet aggregate formation in the lungs, marrow suppression, and immune clearance $[50,51]$. The incidence of thrombocytopenia varies with the severity of the disease $[12,16,50,51]$. Mild thrombocytopenia (a platelet count of $100-150 \times 10^{9} / \mathrm{L}$ ) was detected in $5-41.7 \%$ of patients with COVID-19 infection, $[12,16,50,51]$ and in $58-95 \%$ of patients with severe disease $[16,52,53]$; on average, patients with severe disease had a platelet count of only $23 \times 10^{9} / \mathrm{L}$ to $31 \times 10^{9} / \mathrm{L}$ lower than those with the non-severe disease $[54,55]$. A meta-analysis of 9 studies including 1,779 COVID-19 patients suggested that thrombocytopenia is significantly associated with a threefold increased risk of severe disease and also with the risk of mortality [54]. A further systematic review and meta-analysis of 24 studies involving 5,637 COVID-19 patients suggested that the weighted incidence of thrombocytopenia was $12.4 \%$ and was associated with the severity and outcome of COVID-19 [56].

Many studies have shown that several inflammatory parameters and cytokines were significantly elevated in severe patients with COVID-19 or those admitted to the ICU [11,20,41]. Among the inflammatory parameters, CRP, erythrocyte sedimentation rate (ESR), procalcitonin (PCT), and ferritin were commonly elevated in the COVID-19 infection, but with varying values.

CRP is one of the most distinctive acute phase reactants that can increase rapidly following inflammation, cell damage, or tissue injury. Elevated CRP level is associated with a poor prognosis of COVID-19 infection. Among 78 patients with COVID-19 infection, the CRP was significantly elevated in the progression group compared to the improvement/stabilization group (38.9 versus $10.6 \mathrm{mg} / \mathrm{L}, \mathrm{P}=0.024$ ) [57]. Another study of $298 \mathrm{pa-}$ tients with COVID-19, found that admission CRP level correlated with the severity of disease and predicted an adverse outcome [58]. With a cut-off value of 41.3 , CRP demonstrated a sensitivity of $65 \%$, specificity of $83.7 \%$, for discrimination of disease severity, with the area under the curve (AUC) of the receiver-operating characteristic 
(ROC) of 0.783 . More notably, the positive predictive value (PPV) was $81.6 \%$, indicating that $81.6 \%$ of COVID-19 patients will develop severe disease. CRP was also a good independent predictor of adverse outcomes with an AUC of 0.833 , a sensitivity of $90.5 \%$, a specificity of $77.6 \%$, PPV of $61.3 \%$, and negative predictive value (NPV) of $95.4 \%[58]$.

ESR is another measure of acute-phase reactants that reflects an inflammatory condition but lacks specificity such as CRP [59]. Marked ESR increases (>100 $\mathrm{mm} / \mathrm{hr}$ ) are commonly associated with certain infection (33\%), neoplasm (17\%), and end-stage renal disease (17\%) [59]. A high level of ESR is also found in severe COVID-19 patients [21].

Many studies have shown that elevated PCT levels are significantly associated with the severity of COVID-19 infection $[11,16,20,46,60]$. A meta-analysis also showed that increased PCT level was associated with an approximately 5-fold higher risk of severe COVID-19 infection (OR 4.76) [61]. Additionally, serial PCT measurements may be contributed to predicting progression towards a more severe form of the disease [60]. A study in 95 patients with COVID-19 infection showed that the PCT level was decreased during recovery in discharged patients and there was a significant difference between the levels within 3 days of admission and 7 days before hospital discharge, while the PCT level increased as the disease progressed in non-survivor cases [60]. Of note, a substantial increase in PCT levels may indicate bacterial coinfection in COVID-19 patients who have developed severe form of disease. A study of 41 patients with COVID-19, showed that increased PCT levels were $25 \%$ versus $0 \%$ in ICU patients compared to non-ICU patients, respectively. All patients in the ICU had secondary infections [20].

Ferritin level was reported to be significantly higher in COVID-19 patients with severe/critical disease and was the last laboratory value to return to normal [62]. On the other hand, hs-CRP returned to normal levels at least 5 days before ferritin [62]. It also reported the increase in ferritin level is associated with the worsening of the COVID-19 infection [31]. A systematic review and meta-analysis of 52 studies involving 10,614 confirmed COVID-19 patients showed a significant increase in ferritin levels in severe patients compared to non-severe patients and a significant increase in ferritin levels in non-survivors compared to survivors [63]. In patients with one or more comorbidities, including diabe- tes, thrombotic complications, and cancer, ferritin levels were also significantly higher [63]. Ferritin is thus associated with poor prognosis and could predict worsening of COVID-19 patients [63].

Increased lactate dehydrogenase level (LDH) was also a common laboratory finding among COVID-19 patients [21]. Among 99 patients with COVID-19 infection, 76\% showed elevated LDH levels [21]. Elevated LDH levels are commonly observed in COVID-19 patients with severe disease compared to those with non-severe disease, and they are also associated with an increased risk of death [64]. A pooled analysis of 9 studies, including 1,532 patients with COVID-19, showed that increased LDH levels were associated with an approximately 6-fold increase in the probability of having severe disease and an approximately 16-fold increase in odds of mortality [64]. In another retrospective study of 191 patients with COVID-19, non-survivors, as compared with survivors, had significantly higher levels of LDH, PCT, serum ferritin, and IL-6 [31]. It has also been shown that an increase or decrease in LDH is considered to be an indicator of radiographic progression or improvement as an increase in LDH at a cut-off value of $62.5 \mathrm{U} / \mathrm{L}$ significantly predicted progression of CT chest image while LDH at a cut-off value of $48.5 \mathrm{U} / \mathrm{L}$ predicted an improvement in CT chest image [65].

Increased levels of numerous inflammatory cytokines; prominent among them are IL-6, IL-2, IL-7, IL-10, granulocyte-colony stimulating factor, interferon- $\gamma($ IFN- $\gamma$ )-inducible protein, monocyte chemoattractant protein, macrophage inflammatory protein $1 \alpha$, and TNF- $\alpha$ were detected in many critical COVID-19 patients admitted to the ICU, which suggested that a cytokine storm occurred and contributed to the severity and prognosis of the disease $[20,21]$.

In a retrospective study of 54 patients with critical or severe COVID-19 infection, inflammatory cytokines including IL-1, soluble IL-2 receptor [sIL-2R], IL-6, IL-8, IL-10, and TNF- $\alpha$ were evaluated in 47 patients within 24 hours of admission [62]. The study found that the levels of IL- 6 and sIL-2R in critically ill patients were significantly higher than those with severe disease, whereas the levels of IL-10 and TNF- $\alpha$ levels did not differ significantly between the two groups [62].

Elevated D-dimer and other coagulation parameters abnormalities such as prothrombin time (PT), activated partial thromboplastin time (aPTT) international normalized ratio (INR), thrombin time (TT), fibrinogen degradation products, in COVID-19 patients 
have been associated with a higher risk of acute respiratory distress syndrome (ARDS), ICU admission or death [20,21,31,66,67]. A study of 1,561 patients with COVID-19 infection in China showed that patients with severe COVID-19 have significantly abnormal coagulation parameters including PT, aPTT, INR, TT, fibrinogen degradation products, D-dimer [68]. Besides, a meta-analysis including the previous study and other 12 studies involving a total of 2,574 patients with COVID-19 infection showed that the odds ratio of severe COVID-19 was associated with D-dimer level higher than $0.5 \mu \mathrm{g} / \mathrm{ml}$ on admission [68]. Furthermore, elevated D-dimer level greater than $1 \mu \mathrm{g} / \mathrm{mL}$ in COVID-19 patients, on admission was associated with increased mortality $[31,66]$.

High levels of creatine Kinase, alanine aminotransferase, aspartate aminotransferase, total bilirubin, urea, creatinine and decreased levels of albumin have also been observed in COVID-19 patients $[16,26]$.

Notably, none of the previous laboratory features are specific to COVID-19. Thus the diagnosis should be confirmed by molecular and serological investigations of SARS-CoV-2, while initial treatment would be focused on the clinical and epidemiological evaluation of the risk of COVID-19 infection [19].

\section{Radiologic findings}

Chest computed tomography (CT) abnormalities of COVID-19 are usually ground-glass opacity (GGO) with or without consolidation combined with bilateral, multilobe, peripheral, posterior, and diffuse or lower involvement of the lung $[24,69]$.

Typical patterns of GGOs in COVID-19 include crazy-paved, rounded, or linear morphology [24]. A crazy-paving pattern is defined as an inter- and intralobular septal thickening superimposed on diffuse GGO [24]. Solid pulmonary nodules, cavitation, and mediastinal/hilar lymph node enlargement are not usually observed in COVID-19 [69]. Pleural effusions are unusual [69]. Linear consolidations and other signs indicating organizing pneumonia such as the reverse halo sign (i.e., areas of GGO surrounded by a complete or almost complete ring of consolidation) are also seen several days after the onset of disease [69]. A literature review of COVID-19 infection in neonates and children has shown that the GGO was less frequent at hospital admission (32.7\%) but, it was more frequent in patients admitted to the Pediatric Intensive Care Unit (PICU) for respiratory failure [44].

According to the Fleischner Society for thoracic radiology, indications of chest CT imaging in COVID-19 include patients with moderate/severe symptoms irrespective of RT-PCR test results, evidence of deterioration of respiratory status as indicated by hypoxemia [70]. Normal chest CT scans have been observed in many patients with COVID-19 infection at an early stage of infection [69]. In the previous study of 1,099 patients with COVID-19 infection from China, no radiographic or CT abnormality was reported in $17.9 \%$ (157 of 877 ) patients with non-severe disease and in $2.9 \%$ (5 of 173) patients with severe disease [16]. On the other hand, abnormal chest CT findings may develop in asymptomatic patients [69]. A study of 295 confirmed cases with COVID-19 showed that 49 cases had an initial negative CT scan and 15/49 developed findings after 3-6 days, while in 34/49 cases, CT scans remained negative[71]. Of note, CT imaging is not routinely recommended as a COVID-19 screening test in asymptomatic individuals, and patients with mild COVID-19 symptoms unless they are at risk of disease progression [70]. Impotently, COVID-19 testing is essential to confirm any suspected infection based on imaging findings [70]. Chest CT imaging without confirmation by RT-PCR has limited specificity for the identification of COVID-19 [72]. In a retrospective study of 1,014 suspected COVID-19 cases in China, chest CT findings were compared with RT-PCR. Of the total patients, 59\% (601/1014) had positive RT-PCR results, and $88 \%(888 / 1,014)$ had a positive chest CT scan. Based on positive RT-PCR results, chest CT imaging had higher sensitivity of $97 \%$ (580/601) and a specificity of only $25 \%$ (105/413). The PPV, NPV and accuracy were 65\% (580/888) 83\% (105/126), and 68\% respectively [72].

CT abnormalities of COVID-19 pneumonia vary over time, with different presentations depending on the stage and severity of the lung infection. Pan et al. classified the evolution of lung features into four stages based on time during recovery from COVID-19 (Table 3) [24].

The extent of CT abnormalities has been visually quantified. Each of the five lung lobes was visually graded from 0 to 5 as follows: 0 , no involvement; $1,<5 \%$ involvement; $2,25 \%$ involvement; 3, 26\%-49\% involvement; 4, 50\%-75\% involvement; and 5 , $>75 \%$ involvements [24].

Based on the total CT score, the absorption stage was extended for 26 days from the onset of the initial symptoms [24].

Initial CXR may be normal [70] and may lack the sensitivity to identify some of the lung abnormalities frequently observed in mild or early COVID-19 pneumonia that are otherwise recognized by chest CT $[16,19,70]$. Similar to CT, the most widely reported 


\begin{tabular}{|c|c|c|}
\hline Stage & Time of onset & Main CT imaging features \\
\hline Early stage & $\begin{array}{c}\text { 0-4 days after the onset of } \\
\text { initial symptoms }\end{array}$ & Normal CT findings or mainly GGO. \\
\hline Progressive stage & $\begin{array}{c}\text { D-8 days after symptomatic } \\
\text { presentation }\end{array}$ & Diffuse GGO, crazy-paving appearance, and consolidation. \\
\hline Peak stage & $\begin{array}{c}\text { 9-13 days after symptomatic } \\
\text { presentation }\end{array}$ & $\begin{array}{c}\text { Diffuse GGO, crazy-paving appearance, consolidation, and residual parenchymal } \\
\text { bands. }\end{array}$ \\
\hline Late stage & $\begin{array}{c}\geq 14 \text { days after symptomatic } \\
\text { presentation }\end{array}$ & $\begin{array}{c}\text { Gradual absorption of consolidation and ground-GGO, disappearance of crazy- } \\
\text { paving pattern due to recovery, whereas signs of fibrosis (including parenchy- } \\
\text { mal bands, architectural distortion, and traction bronchiectasis) may appear. }\end{array}$ \\
\hline
\end{tabular}

Table 3: Chest CT Evaluation in COVID-19 [24]

CXR findings in COVID-19 include GGOs and lung consolidation $[21,26,70,73]$. CXR findings are likely to peak at 6 - 12 days of symptoms onset of COVID-19 pneumonia [73]. CXR may be preferred over CT when access to CT is limited unless features of respiratory worsening indicate the use of CT [70]. To reduce the risk of cross-infection of COVID-19 from moving patients, some centers request only the anterior-posterior CXR (usually bedside) to be performed $[70,73]$.

\section{Conclusion}

The COVID-19 pandemic continues to spread worldwide at an alarming rate. This review tried to discuss the current literature on the transmission, clinical features, common laboratory as well as radiologic findings of COVID-19 infection.

Human-to-human transmission occurs mainly through the respiratory route.

The symptoms of COVID-19 range from asymptomatic or mild infection to critical illness. COVID-19 is most commonly associated with respiratory symptoms, but it can also cause several extrapulmonary manifestations. The proportion of the transmission of the disease from presymptomatic and asymptomatic individuals compared with transmission from symptomatic patients remains unclear.

Many non-specific laboratory parameters are affected in COVID-19 patients, some of which are associated with disease severity and poor prognosis, such as severe lymphopenia, abnormal co- agulation parameters, and elevated levels of D-dimer, ferritin, and CRP. Typical CT features of COVID-19 pneumonia include multiple GGO with or without consolidation. Less typical features are also described. CT abnormalities vary over time, with different presentations based on the stage and severity of the lung infection.

\section{Financial Support}

None.

\section{Declaration of Competing Interest}

None.

\section{Bibliography}

1. Gorbalenya A E., et al. "The species Severe acute respiratory syndrome-related coronavirus: classifying 2019-nCoV and naming it SARS-CoV-2". Nature Microbiology 5.4 (2020): 536.

2. Lu H., et al. "Outbreak of pneumonia of unknown etiology in Wuhan, China: The mystery and the miracle". Journal of Medical Virology 92.4 (2020): 92.

3. Worldometer. "Coronavirus Update (Live): COVID-19 Virus Pandemic". Worldometer (2020).

4. World Health Organization. "Transmission of SARS-CoV-2: implications for infection prevention precautions. Scientific brief, 09 July 2020". WHO (2020): 1-10.

5. European Centre for Disease Prevention and Control. "Questions and answers on COVID-19: Basic facts". ECDC (2020). 
6. Centers for Disease Control and Prevention. "How COVID-19 Spreads | CDC". CDC (2020).

7. World Health Organization. "Report of the WHO-China Joint Mission on Coronavirus Disease 2019 (COVID-19)". WHO (2020): 16-24.

8. Qin J., et al. "Estimation of incubation period distribution of COVID-19 using disease onset forward time: A novel crosssectional and forward follow-up study". Science Advances 6.33 (2020): eabc1202.

9. da Rosa Mesquita R., et al. "Clinical manifestations of COVID-19 in the general population: systematic review". Wiener klinische Wochenschrift (2020).

10. Center for Disease Control. "Coronavirus (COVID-19) frequently asked questions | CDC". CDC (2020).

11. Wang D., et al. "Clinical Characteristics of 138 Hospitalized Patients with 2019 Novel Coronavirus-Infected Pneumonia in Wuhan, China". JAMA - Journal of the American Medical Association 323.11 (2020): 1061-1069.

12. Yang X., et al. "Thrombocytopenia and its association with mortality in patients with COVID-19". Journal of Thrombosis and Haemostasis 18.6 (2020): 1469-1472.

13. Bhatraju PK., et al. "Covid-19 in Critically Ill Patients in the Seattle Region - Case Series". New England Journal of Medicine 382.21 (2020): 2012-2022.

14. Liu Y., et al. "Viral dynamics in mild and severe cases of COVID-19". The Lancet Infectious Diseases 20.6 (2020): 656-657.

15. Gupta A., et al. "Extrapulmonary manifestations of COVID-19". Nature Medicine 26.7 (2020): 1017-1032.

16. Guan W., et al. "Clinical Characteristics of Coronavirus Disease 2019 in China". New England Journal of Medicine 382.18 (2020): 1708-1720.

17. Stokes EK., et al. "Coronavirus Disease 2019 Case Surveillance - United States, January 22-May 30, 2020". MMWR Morbidity and Mortality Weekly Report 69.24 (2020): 759-765.

18. Grant MC., et al. "The prevalence of symptoms in 24,410 adults infected by the novel coronavirus (SARS-CoV-2; COVID-19): A systematic review and meta-analysis of 148 studies from 9 countries". Hirst JA, editor. PLOS ONE 15.6 (2020): e0234765.
19. Wong CKH., et al. "Clinical presentations, laboratory and radiological findings, and treatments for 11,028 COVID-19 patients: a systematic review and meta-analysis". Scientific Reports 10.1 (2020): 19765.

20. Huang C., et al. "Clinical features of patients infected with 2019 novel coronavirus in Wuhan, China". The Lancet 395.10223 (2020): 497-506.

21. Chen N., et al. "Epidemiological and clinical characteristics of 99 cases of 2019 novel coronavirus pneumonia in Wuhan, China: a descriptive study". The Lancet 395.10223 (2020): 507-513.

22. Richardson S., et al. "Presenting Characteristics, Comorbidities, and Outcomes Among 5700 Patients Hospitalized With COVID-19 in the New York City Area". JAMA 323.20 (2020): 2052.

23. Yang BY., et al. "Clinical Characteristics of Patients With Coronavirus Disease 2019 (COVID-19) Receiving Emergency Medical Services in King County, Washington". JAMA Network Open 3.7 (2020): e2014549.

24. Pan F., et al. "Time Course of Lung Changes at Chest CT during Recovery from Coronavirus Disease 2019 (COVID-19)". Radiology 295.3 (2020): 715-721.

25. Liu K., et al. "Clinical characteristics of novel coronavirus cases in tertiary hospitals in Hubei Province". Chinese Medical Journal 133.9 (2020): 1025-1031.

26. Zhang G., et al. "Clinical features and short-term outcomes of 221 patients with COVID-19 in Wuhan, China". Journal of Clinical Virology 127 (2020): 104364.

27. Chen J., et al. "Clinical progression of patients with COVID-19 in Shanghai, China”. Journal of Infection 80.5 (2020): e1-6.

28. Li R., et al. "Substantial undocumented infection facilitates the rapid dissemination of novel coronavirus (SARS-CoV-2)". Science 368.6490 (2020): 489-493.

29. Norman DC and Yoshikawa TT. "Fever in the elderly". Infectious disease clinics of North America 10.1 (2021): 93-99.

30. Arons MM., et al. "Presymptomatic SARS-CoV-2 Infections and Transmission in a Skilled Nursing Facility". New England Journal of Medicine 382.22 (2020): 2081-2090. 
31. Zhou F., et al. "Clinical course and risk factors for mortality of adult inpatients with COVID-19 in Wuhan, China: a retrospective cohort study". The Lancet 395.10229 (2020): 1054-1062.

32. Liang W., et al. "Development and validation of a clinical risk score to predict the occurrence of critical illness in hospitalized patients with COVID-19". JAMA Internal Medicine 180.8 (2020): 1081-1089.

33. Kaeuffer C., et al. "Clinical characteristics and risk factors associated with severe COVID-19: prospective analysis of 1,045 hospitalised cases in North-Eastern France, March 2020". Euro surveillance: bulletin Europeen sur les maladies transmissibles $=$ European Communicable Disease Bulletin 25.48 (2020): 2000895.

34. Pan L., et al. "Clinical characteristics of COVID-19 patients with digestive symptoms in Hubei, China: A descriptive, crosssectional, multicenter study" American Journal of Gastroenterology 115.5 (2020): 766-773.

35. Goyal P., et al. "Clinical Characteristics of Covid-19 in New York City”. New England Journal of Medicine 382.24 (2020): 23722374.

36. Cheung KS., et al. "Gastrointestinal Manifestations of SARSCoV-2 Infection and Virus Load in Fecal Samples From a Hong Kong Cohort: Systematic Review and Meta-analysis". Gastroenterology 159.1 (2020): 81-95.

37. Tong JY., et al. "The Prevalence of Olfactory and Gustatory Dysfunction in COVID-19 Patients: A Systematic Review and Meta-analysis". Otolaryngology-Head and Neck Surgery 163.1 (2020): 3-11.

38. Giacomelli A., et al. "Self-reported olfactory and taste disorders in patients with severe acute respiratory coronavirus 2 infection: A cross-sectional study". Clinical Infectious Diseases. Oxford University Press 71 (2020): 889-890.

39. Yang X., et al. "Clinical course and outcomes of critically ill patients with SARS-CoV-2 pneumonia in Wuhan, China: a singlecentered, retrospective, observational study". The Lancet Respiratory Medicine 8.5 (2020): 475-481.

40. Lechien JR., et al. "Loss of Smell and Taste in 2013 European Patients With Mild to Moderate COVID-19". Annals of Internal Medicine NLM (Medline) 173 (2020): 672-675.

41. Wu C., et al. "Analysis of therapeutic targets for SARS-CoV-2 and discovery of potential drugs by computational methods". Acta Pharmaceutica Sinica B 10.5 (2020): 766-788.
42. Centers for Disease Control and Prevention. "COVID-19 in children and teens". CDC (2020).

43. Viner RM., et al. "Systematic review of reviews of symptoms and signs of COVID-19 in children and adolescents". Archives of Disease in Childhood (2020): archdischild-2020-320972.

44. Di Nardo M., et al. "A literature review of 2019 novel coronavirus (SARS-CoV2) infection in neonates and children". Pediatric Research (2020): 1-8.

45. Liguoro I., et al. "SARS-COV-2 infection in children and newborns: a systematic review". European Journal of Pediatrics 179.7 (2020): 1029-1046.

46. Zhang J jin., et al. "Clinical characteristics of 140 patients infected with SARS-CoV-2 in Wuhan, China”. Allergy: European Journal of Allergy and Clinical Immunology 75.7 (2020): 17301741.

47. Huang I and Pranata R. "Lymphopenia in severe coronavirus disease-2019 (COVID-19): Systematic review and meta-analysis". Journal of Intensive Care. BioMed Central Ltd. 8 (2020): 36.

48. Terpos E., et al. "Hematological findings and complications of COVID-19". American Journal of Hematology. Wiley-Liss Inc 95 (2020): 834-847.

49. Liu Y., et al. "Neutrophil-to-lymphocyte ratio as an independent risk factor for mortality in hospitalized patients with COVID-19". Journal of Infection 81.1 (2020): e6-12.

50. Zhang Y., et al. "Mechanisms involved in the development of thrombocytopenia in patients with COVID-19". Thrombosis Research 193 (2020): 110-115.

51. Wool GD and Miller JL. "The Impact of COVID-19 Disease on Platelets and Coagulation". Pathobiology 88.1 (2021): 15-27.

52. Levi M., et al. "Coagulation abnormalities and thrombosis in patients with COVID-19". The Lancet Haematology. Elsevier Ltd 7 (2020): e438-40.

53. Thachil J. "What do monitoring platelet counts in COVID-19 teach us?". Journal of Thrombosis and Haemostasis : JTH 18.8 (2020): 2071-2072.

54. Lippi G., et al. "Thrombocytopenia is associated with severe coronavirus disease 2019 (COVID-19) infections: A meta-analysis". Clinica Chimica Acta 506 (2020): 145-148. 
55. Henry BM., et al. "Hematologic, biochemical and immune biomarker abnormalities associated with severe illness and mortality in coronavirus disease 2019 (COVID-19): a metaanalysis"Clinical Chemistry and Laboratory Medicine (CCLM) 58.7 (2020): 1021-1028.

56. Zong X., et al. "Thrombocytopenia Is Associated with COVID-19 Severity and Outcome: An Updated Meta-Analysis of 5637 Patients with Multiple Outcomes". Laboratory Medicine 52.1 (2021): 10-15.

57. Liu W., et al. "Analysis of factors associated with disease outcomes in hospitalized patients with 2019 novel coronavirus disease". Chinese Medical Journal 133.9 (2020): 1032-1038.

58. Luo X., et al. "Prognostic Value of C-Reactive Protein in Patients With Coronavirus 2019". Clinical Infectious Diseases : An Official Publication of the Infectious Diseases Society of America 71.16 (2020): 2174-2179.

59. Fincher RME and Page MI. "Clinical Significance of Extreme Elevation of the Erythrocyte Sedimentation Rate". Archives of Internal Medicine 146.8 (1986): 1581-1583.

60. Hu R., et al. "Procalcitonin levels in COVID-19 patients". International Journal of Antimicrobial Agents 56.2 (2020).

61. Lippi G and Plebani M. "Procalcitonin in patients with severe coronavirus disease 2019 (COVID-19): A meta-analysis". Clinica Chimica Acta 505 (2020): 190-191.

62. Li Y., et al. "Retrospective analysis of laboratory testing in 54 patients with severe- or critical-type 2019 novel coronavirus pneumonia". Laboratory Investigation 100.6 (2020): 794-800.

63. Cheng L., et al. "Ferritin in the coronavirus disease 2019 (COVID-19): A systematic review and meta-analysis". Journal of Clinical Laboratory Analysis. John Wiley and Sons Inc 34 (2020).

64. Henry BM., et al. "Lactate dehydrogenase levels predict coronavirus disease 2019 (COVID-19) severity and mortality: A pooled analysis". American Journal of Emergency Medicine 38.9 (2020): 1722-1726.

65. Wu M-Y., et al. "Clinical evaluation of potential usefulness of serum lactate dehydrogenase (LDH) in 2019 novel coronavirus (COVID-19) pneumonia". Respiratory Research 21.1 (2020): 171.
66. Tang N., et al. "Abnormal coagulation parameters are associated with poor prognosis in patients with novel coronavirus pneumonia". Journal of Thrombosis and Haemostasis 18.4 (2020): 844-847.

67. Wu C., et al. "Risk Factors Associated With Acute Respiratory Distress Syndrome and Death in Patients With Coronavirus Disease 2019 Pneumonia in Wuhan, China". JAMA Internal Medicine 180.7 (2020): 934-943.

68. Yu HH., et al. "D-dimer level is associated with the severity of COVID-19”. Thrombosis Research 195 (2020): 219-225.

69. Ng M-Y., et al. "Imaging Profile of the COVID-19 Infection: Radiologic Findings and Literature Review". Radiology: Cardiothoracic Imaging 2.1 (2020): e200034.

70. Rubin GD., et al. "The role of chest imaging in patient management during the covid-19 pandemic: A multinational consensus statement from the fleischner society". Radiology 296.1 (2020): 172-180.

71. Ling Z., et al. "Asymptomatic SARS-CoV-2 infected patients with persistent negative CT findings". European Journal of Radiology. Elsevier Ireland Ltd 126 (2020).

72. Ai T., et al. "Correlation of Chest CT and RT-PCR Testing for Coronavirus Disease 2019 (COVID-19) in China: A Report of 1014 Cases". Radiology 296.2 (2020): E32-40.

73. Jacobi A., et al. "Portable chest X-ray in coronavirus disease-19 (COVID-19): A pictorial review". Clinical Imaging. Elsevier Inc 64 (2020): 35-42.

\section{Assets from publication with us}

- Prompt Acknowledgement after receiving the article

- Thorough Double blinded peer review

- Rapid Publication

- Issue of Publication Certificate

- High visibility of your Published work

Website: https://www.actascientific.com/

Submit Article: https://www.actascientific.com/submission.php Email us: editor@actascientific.com

Contact us: +919182824667 